Radiologe 2010 · 50:208

DOI 10.1007/s00117-010-1983-z

Online publiziert: 21. Februar 2010

(c) Springer-Verlag 2010

\author{
C.J.Zech • M.F. Reiser \\ Institut für Klinische Radiologie, Klinikum der \\ Ludwig-Maximilians-Universität München, Campus Großhadern, München
}

\title{
Das akute Abdomen
}

\section{Eine interdisziplinäre Herausforderung}

Sehr geehrte Leserinnen und Leser,

der medizinische Notfall stellt immer eine besondere Herausforderung für die behandelnden Ärztinnen und Ärzte dar. Bei häufig eingeschränkter personeller Besetzung muss in Notfall- und Bereitschaftsdiensten zu jeder Tages- und Nachtzeit individuell, rasch und kompetent ein wirkungsvolles und zielführendes therapeutisches Konzept entwickelt werden. Für den Symptomkomplex des akuten Abdomens schwankt die richtige Behandlungsstrategie von „watch and wait", über medikamentöse Therapien bis hin zur Notfalloperation oder -intervention. Diese Entscheidung basiert auf der richtigen Diagnose - hier ist die bildgebende Diagnostik neben der Anamnese und körperlichen Untersuchung sowie der Labordiagnostik der tragende Pfeiler der diagnostischen Abklärung.

Die Ursachen eines akuten Abdomens könnten vielfältiger nicht sein. Letztlich können Erkrankungen aller abdomineller Organsysteme einschließlich deren Gefäßversorgung ein akutes Abdomen verursachen. Am häufigsten sind entzündliche Erkrankungen der Bauchorgane, gefolgt von mechanischen Ursachen wie Obstruktion oder Perforation von Hohlorganen oder Blutgefäßen. Diese Vielfalt an Differenzialdiagnosen erklärt auch die große Herausforderung, die damit für den Radiologen verbunden ist. Dieser muss an Hand der zum Zeitpunkt der Diagnostik vorliegenden Befunde die richtige Modalität und Untersuchungstechnik wählen, um dann zur korrekten Diagnose zu kommen. Für diese Entscheidungsfindung ist die Abdomensonographie häufig unabdingbar, die nicht zuletzt aus diesem Grund ein integraler Bestandteil aller üblichen Diagnosealgorithmen beim akuten Abdomen ist. Der Sonographie ist aus diesem Grund auch ein übergeordneter Artikel in diesem Themenheft von „Der Radiologe" gewidmet.

Die Computertomographie hat sich in den letzten Jahren zum wichtigsten bildgebenden Instrument beim akuten Abdomen entwickelt und stellt deshalb einen Schwerpunkt der einzelnen Beiträge dar. Sie sollte immer dann zum Einsatz kommen, wenn die Diagnose durch die Sonographie nicht sicher gestellt werden kann oder wenn wegen der Erkrankungsschwere bzw. wegen der Dringlichkeit des geplanten operativen oder interventionellen Eingriffs eine besonders sorgfältige und gut dokumentierbare Indikationsstellung notwendig ist. Dies trifft im klinischen Alltag eines Großklinikums wie dem Klinikum der Universität München regelmäßig und zu jeder Tages- und Nachtzeit zu und wird sicherlich auch so in Krankenhäusern jeder Größe mit einer Notfallambulanz erlebt. Die Übersichtsaufnahme des Abdomens ist demgegenüber deutlich in den Hintergrund getreten.

Die rechtfertigende Indikation hat das potenzielle Risiko einer Strahlenexposition gegenüber dem Nutzen für die Gesundheit des jeweiligen Patienten kritisch abzuwägen. Diese Abwägung hat sich an evidenzbasierten wissenschaftlichen Kriterien zu orientieren. Der höchste Evidenzgrad wird mit doppelblinden randomisierten Studien erreicht und hat das Langzeitüberleben als Endpunkt der Analyse zu bewerten. Dieser Forderung ist aus ethischen Gründen bei Verfahren, die ihren praktischen klinischen Nutzen auf breiter Front bewiesen haben, kaum zu entsprechen. Das wäre genauso bizarr, als wollte man eine Studie durchführen, bei der die Röntgenaufnahme des Schädels mit der kranialen CT bei der Versorgung von Patienten mit schwerem Schädel-Hirn-Trauma hinsichtlich des Outcomes für die Patienten verglichen wird. Gleichwohl ist darauf zu achten, dass die CT auch bei Notfallindikationen, wie dem akuten Abdomen, mit möglichst dosisoptimierten Protokollen durchgeführt wird.

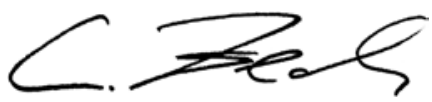

Dr. Christoph J. Zech

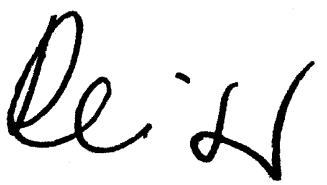

Prof. Dr. Dr. h.c. Maximilian F. Reiser 